\title{
Phase transition induced by a magnetic field
}

\author{
G.W. Semenoff ${ }^{1}$, I.A. Shovkovy ${ }^{2 *}$, and L.C.R. Wijewardhana ${ }^{2}$ \\ ${ }^{1}$ Department of Physics and Astronomy, \\ University of British Columbia \\ 6224 Agricultural Road, Vancuver, \\ British Columbia V6T 1Z1, Canada \\ ${ }^{2}$ Physics Department, University of Cincinnati \\ Cincinnati, Ohio 45221-0011, USA
}

March 20, 2018

\begin{abstract}
The magnetic catalysis of discrete chiral symmetry breaking in the $2+1$ dimensional Nambu-Jona-Lasinio model is analyzed. A particular attention is paid to a possible application of the effect in solid state physics. The fermion contribution to the thermal conductivity as a function of the dynamical mass (energy gap in the spectrum) is estimated and is shown to be suppressed when a non-zero order parameter develops.
\end{abstract}

\footnotetext{
*On leave of absence from Bogolyubov Institute for Theoretical Physics, Kiev 252143, Ukraine
} 
Chiral symmetry breaking induced by an external magnetic field has recently been studied in a few $2+1$ and $3+1$ dimensional relativistic field theories 11, 2, 3, 4, 5] (for some earlier results on the subject see Refs. [6, 8, 8]). In particular, a magnetic field enhances fermion condensation in the Nambu-Jona-Lasinio (NJL) model in $2+1$ dimensions. The solution of the gap equation indicates that the critical 4-Fermi coupling required to generate a chiral symmetry breaking condensate with $\langle\bar{\psi} \psi\rangle \neq 0$ goes to zero as soon as the magnetic field is switched on. In fact, there are two branches of the solution depending on whether the effective 4-Fermi coupling $g$ is less than or greater than $g_{c}$, where $g_{c}$ is the critical coupling in the NJL model at zero magnetic field. In the subcritical region $g<g_{c}$ the non-trivial symmetry breaking solution disappears as the field goes to zero. On the other hand, at $g>g_{c}$ the solution goes to the usual symmetry breaking solution in the NJL model without a magnetic field.

In this letter we promote the hypothesis that this magnetic catalysis of chiral symmetry breaking can be used to explain recent experimental results of K. Krishana et al [9]. They observed a magnetic field induced phase transition in a high- $\mathrm{T}_{c}$ superconductor (below $T_{c}$ ). The experiment shows that the thermal conductivity as a function of temperature and magnetic field displays a sharp break in its slope. There are several competing arguments which attempt to explain this behavior. In one line of reasoning, the behavior is accounted for the formation of an energy gap at the Fermi surface. In d-wave superconductors there is no energy gap in the spectrum of the quasi-electrons. The Fermi surface consists of four independent degeneracy points. The gap (as well as the temperature of the transition) as a function of field strength follows a square root law over a large range of fields. We present this as an alternative to the work in [10] which deals with some general scaling properties of a model in a magnetic field, the work of Laughlin [11] which relates the phase transition to the appearance of an additional parity and time-reversal symmetry violating superconducting order parameter which may develop under specific conditions 112 and other speculations that the behavior is a signal of a phase transition in the lattice of magnetic flux lines. While our work was in progress, we became aware of a paper by K. Farakos and N.E. Mavromatos 13 where a similar attempt of applying the magnetic catalysis to the explanation of the phase transition observed by Krishana et al [9] was made. They considered the magnetic catalysis in a gauge theory with a continuous symmetry, while we study the NJL model with discrete symmetry. We also estimate the fermion contribution to the thermal conductivity as a function of the dynamical mass, and show that it is suppressed when the order parameter develops a nonzero expectation value.

In our hypothesis, the additional order parameter which signals the phase transition is a neutral condensate of quasi-electron-hole pairs around the nodes on the Fermi surface in a d-wave superconductor. The distinctive feature is the neutrality of the condensate, and the parity as well as time-reversal symmetry conserving character of the order parameter (same as in 13]). The phase transition itself is of second order. 
The NJL model is a natural candidate for the description of the interactions of quasi-electrons in a d-wave superconductor. It is well-known that, when the Fermi level is concentrated at points, the spectrum of the quasi-electrons with energies close to the Fermi level is linear in the momentum $E(\vec{k}) \sim|\vec{k}|$ and can be described by a Dirac Hamiltonian 13, 14, 15. The effective action from which the Dirac Hamiltonian can be derived is the Lorentz invariant Dirac action with two species of 4-component spinors. The two species arise from the existence of four inequivalent degeneracy points on the Fermi surface. The Dirac action has apparent continuous chiral and flavor symmetry which are not a symmetries of the original system which is defined on a lattice.

The interaction term is a 4-Fermi operator. The choice of suitable operators is dictated by the symmetries of the theory. Though the lattice theory does not have continuous chiral symmetry, it does have a symmetry which corresponds to discrete chiral symmetry - invariance under translations by one site on the lattice. Translation by one site interchanges the degeneracy points of the Fermi surface and corresponds to a discrete chiral transformation in the continuum. For this reason it is natural to choose a 4 -Fermi interaction which breaks the continuous chiral symmetry to its discrete $Z_{2}$ subgroup.

It is also natural to choose a 4-Fermi operator which is invariant under parity, time reversal and charge conjugation invariance. Parity and time reversal are symmetries of the theory in the absence of an external magnetic field and chargeconjugation invariance is equivalent to the approximate particle-hole symmetry of the low energy excitations.

Even with these restrictions, there are both Lorentz invariant and noninvariant 4-Fermi operators. The rationale for choosing a Lorentz invariant operator is that, it is a combination of Lorentz invariance and chiral symmetry which forbids an energy gap in the spectrum of the continuum model. If we chose a non Lorentz invariant 4-Fermi operator, radiative corrections would gap the quasi-electron spectrum even without an external magnetic field or spontaneous symmetry breaking. A candidate for generating 4-Fermi interactions of the kind that we choose, $-g(\bar{\psi} \psi)^{2}$ is the effective interaction mediated by optical phonons.

Consider the Nambu-Jona-Lasinio model in $2+1$ dimensions [14, 16]. In $2+1$ dimensions the minimal representation of the Dirac algebra is with $2 \times$ 2 matrices and the minimal Dirac spinor has two components. However, for two component fermions, the mass operator $\bar{\psi} \psi$ is a pseudo-scalar and massive fermions violate parity. If there are two species of fermions, the mass operator $\bar{\psi}_{1} \psi_{1}-\bar{\psi}_{2} \psi_{2}$ is a scalar if, besides the spacetime transformation, the fermion species are interchanged under parity. The other combination, $\bar{\psi}_{1} \psi_{1}+\bar{\psi}_{2} \psi_{2}$ is a pseudoscalar. It is convenient to describe the two species of fermions using a reducible $4 \times 4$ representation if the Dirac algebra [17. The $\gamma$-matrices are:

$$
\gamma^{0}=\left(\begin{array}{ll}
\sigma_{3} & 0 \\
0 & -\sigma_{3}
\end{array}\right), \quad \gamma^{1}=\left(\begin{array}{ll}
i \sigma_{1} & 0 \\
0 & -i \sigma_{1}
\end{array}\right), \quad \gamma^{2}=\left(\begin{array}{ll}
i \sigma_{2} & 0 \\
0 & -i \sigma_{2}
\end{array}\right),
$$


where $\sigma_{i}$ are the Pauli matrices.

Thus, the Lagrangian density of our model is given by

$$
\mathcal{L}=\frac{1}{2}\left[\bar{\Psi}, i \gamma^{\mu} D_{\mu} \Psi\right]+\frac{g}{2}(\bar{\Psi} \Psi)^{2},
$$

where the covariant derivative includes only the vector potential for the external magnetic field:

$$
D_{\mu}=\partial_{\mu}-i e A_{\mu}^{e x t}, \quad A_{\mu}^{e x t}=\left(0,-\frac{B}{2} x_{2}, \frac{B}{2} x_{1}, 0\right) .
$$

To apply the $1 / N$ perturbative expansion we assume that fermions have an additional flavor index $i=1,2, \ldots, N$. In the d-wave superconductor $\mathrm{N}=2$.

The standard mass term, $m \bar{\Psi} \Psi$, as is easy to check, is not invariant under the discrete chiral symmetry

$$
\Psi \rightarrow \gamma_{5} \Psi, \quad \bar{\Psi} \rightarrow-\bar{\Psi} \gamma_{5}
$$

So, it is forbidden in the Lagrangian density. However, due to the magnetic catalysis [1] the discrete symmetry is broken dynamically and as a result, fermions get a dynamical mass. Let us stress that symmetry breaking appears already at an arbitrary small 4 -fermion coupling constant.

The latter fact can be understood by the following argument. Consider the Dirac Hamiltonian in a background magnetic field

$$
H=\left(\begin{array}{ll}
h & 0 \\
0 & h
\end{array}\right)
$$

where

$$
h=i \sigma^{2} D_{1}-i \sigma^{1} D_{2} .
$$

In a background field with magnetic flux $\Phi=\frac{1}{2 \pi} \int B d^{2} x$ an index theorem (an open space version of the Atiyah-Singer Index theorem) and a vanishing theorem imply that there are $[|\Phi|]-1$ solutions of the equation $h \phi=0$ where $[|\Phi|]$ is the largest integer less than $|\Phi|$, and their chirality is given by $\sigma^{3} \phi=\operatorname{sign}(\Phi)([|\Phi|]-1)$. Existence of these zero modes of the Hamiltonian implies degeneracy of the second quantized ground state - the degenerate states correspond to all of the ways that the zero modes could be populated by quasielectrons. Overall charge neutrality implies that half of the zero modes should be populated and half of them should be empty. There are chirally symmetric populations of the zero modes where half of the modes with four component eigenfunctions of $H, \Psi_{+}=\left(\begin{array}{l}\phi \\ 0\end{array}\right)$ and half of the modes with wavefunctions $\Psi_{-}=\left(\begin{array}{l}0 \\ \phi\end{array}\right)$ are occupied. The chiral condensate is $\left\langle\Psi^{\dagger} \gamma^{0} \Psi\right\rangle$, and the average 
condensate $\left\langle\int d^{2} x \Psi^{\dagger} \gamma^{0} \Psi\right\rangle$ vanishes. There are also chirally asymmetric populations of the zero modes, for example, when all of the positive helicity modes $\Psi_{+}$ are filled and the negative helicity modes $\Psi_{-}$are empty. They are degenerate with the chirally symmetric ones.

The degeneracy is resolved by adding the interaction terms in the Hamiltonian and diagonalizing the matrix of first order corrections. In this case, the perturbation given by the 4-Fermi operator $-G / 2(\bar{\Psi} \Psi)^{2}$ resolves the degeneracy by lowering the energy of the states with maximally chirally asymmetric populations. It is also easy to see that a similar argument would not work when there is no magnetic field since in that case there are no zero modes and the density of states at the Fermi surface vanishes like $\left|E-E_{F}\right|$. The excited states which would have to be populated in order to have a non-vanishing condensate have positive energy and therefore there is a finite critical coupling which $g$ must achieve before the 4-Fermi can lower the energy of the chirally asymmetric state below that of the ground state.

Conventionally, the symmetry breaking is found using the effective potential in the large $N$ expansion. The analysis of the model with the Lagrangian density in (2) repeats, up to minor differences appearing due to the absence of NambuGoldstone bosons, the analysis in [1]. We shall skip all of the intermediate calculations and go directly to the gap equation

$$
\mu l=\frac{1}{l m_{d y n}}+\sqrt{2} \zeta\left(\frac{1}{2}, 1+\frac{m_{d y n}^{2} l^{2}}{2}\right)+O(1 / \Lambda) .
$$

Here we introduced the mass scale parameter $\mu \equiv 2 \Lambda\left(g_{c}-g\right) / g g_{c}$ (by definition, $g=N G \Lambda / \pi$ and $\left.g_{c}=\sqrt{\pi}\right)$. In addition, the magnetic length $l \equiv 1 / \sqrt{|e B|}$ is used instead of the magnetic field strength $B$. From now on we assume that the value of cutoff $\Lambda$ is much bigger than all other parameters in the model, so that further we can neglect all corrections of order $1 / \Lambda$ or higher.

The gap equation (7) is written for the "subcritical" value of coupling constant, $g \leq g_{c}$ (where $g_{c}$ is the critical value of coupling in the model without the magnetic field). If we try to go into the supercritical region the parameter $\mu$ changes the sign. In this paper, however, we are not going to study the case when $g>g_{c}$. As is clear, by fixing the dimensionful parameter $\mu$, one already defines the NJL model. As soon as the $\mu$ is fixed, we can talk about strong or weak external magnetic fields applied to the system. The quantitative characteristics of that is the value of dimensionless parameter $\mu l$. Indeed, the vanishing field will correspond to infinitely large value of $\mu l$. While increasing the field, the parameter $\mu l$ gets smaller, and eventually goes to zero when field becomes infinitely strong. Of course, different values of $\mu l$ can also be interpreted from another viewpoint: small parameter $\mu l$ corresponds to near-critical value of coupling constant, while large value of $\mu l$ corresponds to weak coupling.

For our analysis below, the first approach seems to be more appropriate since we are interested in the dependence of dynamical mass on the magnetic field 
strength without changing the "parent" NJL model. It would also allow us to make the analysis almost independent of a specific value of coupling constant.

So, let us consider, two limiting cases of field strength: (i) vanishingly weak field (or rather $\sqrt{|e B|} \ll \mu$ ), and (ii) infinitely large field (or just $\sqrt{|e B|} \gg \mu$ ).

To get the gap equation in the case of weak field, one has to notice that large positive value in the right hand side of (7) is obtained for small values of $m_{d y n} l$ when the first term dominates over the zeta function term. Thus, the approximate gap equation reads

$$
\mu l \simeq \frac{1}{l m_{d y n}},
$$

so that the solution for the dynamical mass is proportional to the magnetic field strength,

$$
m_{d y n}=\frac{|e B|}{\mu} .
$$

Here we would like to note that a simple numerical analysis of the gap equation (71) shows that the solution is indeed close to this asymptotic behavior up to a few percent but only in the region where the ratio $\sqrt{|e B|} / \mu$ is less than around 0.005. If the ratio gets bigger than that deviations are more prominent.

The case of strong magnetic field is slightly more complicated. The first approximation for the gap equation in this case is obtained by substituting zero in the left hand side of the gap equation (7):

$$
0=\frac{1}{l m_{d y n}}+\sqrt{2} \zeta\left(\frac{1}{2}, 1+\frac{m_{d y n}^{2} l^{2}}{2}\right) .
$$

This equation can easily be solved numerically, leading to the result $m_{d y n} l=$ $c_{0} \approx 0.446$. And the solution for the dynamical mass is proportional to the square root of the field,

$$
m_{d y n} \simeq c_{0} \sqrt{|e B|}+c_{1} \mu,
$$

where the second term (with $c_{1}=-0.173$ ) is the next to leading term in Taylor expansion around $\mu l=0$. It appears when the magnetic field strength is large but finite. Even though the solution in (11) was obtained under the assumption $\sqrt{|e B|} \gg \mu$, it should be rather reliable even for values of $\sqrt{|e B|}$ of order $\mu$. In fact, the numerical solution of the gap equation, given in Figure 1, confirms our statement. More than that, as a simple analysis shows, the numerical solution can be well approximated by a the square root dependence in the whole region of magnetic fields except for a small region of weak fields.

Thus, summarizing, the dependence of the dynamical mass on the external magnetic field is linear for very small fields (small in comparison with the inherent scale $\mu$ ), then the dependence gradually approaches the asymptote in 
Eq. (11) when the square root of field gets bigger than $\mu$. The graphical solution is shown in Figure 1.

In view of application the result to the phase transition mentioned in the introduction, we have to require that the actual value of the parameter $\mu$ is small enough in comparison with the square root of the magnetic field while given in the appropriate units. So, that the small linear region of the dynamical mass dependence is not experimentally seen. This assumption is not unnatural at all if we recall that the asymptotic behavior in (11) has a rather wide range of validity, while the linear dependence appears only for really very weak fields.

The gap equation at a finite temperature can also be written right away [1]. However, in that case it becomes really complicated and we are not going to write it down here. The qualitative picture though is clear enough even without solving the equation. In particular, we know that the magnetic field helps symmetry breaking, while temperature works against it. As a result, we have a competition of those two influences. If we keep the value of the field being constant, the critical temperature at which the order parameter (gap in the energy spectrum) disappears is, up to an unimportant numerical factor, proportional to the value of the dynamical mass at zero temperature $m_{d y n}(|e B|)$ [1, 3, 19]. On the other hand, considering the mass as a function of the field, we can invert the equation for the critical temperature, and obtain the critical value of the magnetic field at a given temperature. So, looking for the dependence of the dynamical mass on the field strength at a finite temperature, we will find that the gap stays zero until the value of the field is less than critical, and after field becomes stronger than critical, a non-zero gap develops and grows with increasing the field. Later we will make use of this qualitative picture.

We would like to mention that one may be tempted and try to use the magnetic catalysis of $2+1$ dimensional quantum electrodynamics instead of the NJL model. It is indeed a very attractive idea since, in a rather physical limit of a weak magnetic field (and apparently in range of validity of the large $N$ expansion), the dynamical mass as a function of the magnetic field follows the square root law dependence [20]:

$$
m_{d y n} \simeq \frac{\sqrt{|e B|}}{4 \pi \nu_{0} N} \ln \left(4 \pi \nu_{0} N\right),
$$

where $\nu_{0} \approx 0.14$. Note that this dependence is quite natural from the following point of view. As is well known, in $2+1$ dimensional QED it is $1 / N$ rather than $e^{2}$ that plays the role of the coupling constant [17, 22]. The value of $e^{2}$ is more like an ultraviolet cutoff in the model. Therefore, one effectively is left with a single infrared dimensionful parameter, namely $\sqrt{|e B|}$, and the dynamical mass should be proportional to this only one dimensionful parameter.

While considering quantum electrodynamics one should remember, however, that breaking the continuous symmetry is forbidden in $2+1$ dimensions at any finite temperature. In order to avoid this difficulty one should apply additional 
assumptions, like those in [13] where the realization of the BKT phase is conjectured.

Another thing that can obscure the effect of magnetic catalysis in $2+1$ dimensional QED is the parity violating mass term that seems to be generated due to the induced Chern-Simons photon mass in a magnetic field [23]. Even if the effect of magnetic catalysis does not disappear, the quantitative analysis may somewhat change.

It is possible to give a simple estimate of the thermal conductivity dependence on the magnetic field in the model at hand.

To obtain the expression for the thermal conductivity we apply the familiar method of linear response 24]. There are, however, a few things that we need to mention. First, we work with zero chemical potential, so that the general expression for the conductivity is given in terms of the energy-current correlation function alone. And second, to get a finite thermal conductivity we will need to modify the free fermion Green function in the magnetic field by introducing an effective lifetime of quasiparticles. The latter can be interpreted as a result of fermion scattering at impurities of a superconductor, which is always present in real samples. Such an approximation is presumably good enough for our purposes, since the only thing we are interested in is a change in the value of the thermal conductivity that results from the magnetic catalysis of symmetry breaking and generation of the energy gap.

As we mentioned above, the expression for the thermal conductivity can be given in terms of the energy-current (momentum) correlation function 24]

$$
\kappa_{i j}^{e l}(\omega)=\frac{1}{T V} \int_{0}^{\infty} d t \int_{0}^{\beta} d \lambda T r\left\{\rho_{0} P^{i}(0) P^{j}(t+i \lambda)\right\} e^{-i \omega t},
$$

where $V$ is the volume of the system,

$$
\begin{aligned}
P^{i}(0) & =\frac{i}{2} \int d^{2} x\left(\bar{\psi} \gamma^{0} \partial^{i} \psi-\partial^{i} \bar{\psi} \gamma^{0} \psi\right) \\
P^{j}(t) & =e^{i H t} P^{j}(0) e^{-i H t}
\end{aligned}
$$

and $\rho_{0}$ is the equilibrium density matrix:

$$
\rho_{0}=\frac{1}{Z} e^{-\beta H}, \quad Z=\operatorname{Tr}\left(e^{-\beta H}\right) .
$$

Notice here that the action is invariant under translations even in the presence of a constant external magnetic field, and that the definition of the momentum coincides with that for the theory without an external field. Here we do not include the contribution of the gauge fields to the momentum since we are interested in the fermion component of the thermal conductivity. 
By using the definition in Eq. (13), one can obtain the following expression for the static thermal conductivity of an isotropic system [25, 26, 27]

$$
\kappa^{e l}=-\frac{1}{T V} \operatorname{Im} \int_{0}^{\infty} t d t \operatorname{Tr}\left\{\rho_{0} P^{i}(t) P^{i}(0)\right\} .
$$

Let us introduce the following thermal Green function:

$$
\begin{aligned}
G(\tau) & =\frac{1}{V} \operatorname{Tr}\left(\rho_{0} e^{\tau H} P^{i} e^{-\tau H} P^{i}\right), \\
G\left(i \nu_{m}\right) & =\int_{0}^{\beta} G(\tau) e^{i \nu_{m} \tau} d \tau,
\end{aligned}
$$

where $\nu_{m}=2 \pi T m$. It is this Green function $G\left(\nu_{m}\right)$ that can perturbatively be calculated in a theory. The simplest Feynman diagram gives

$$
G\left(p=0, i \nu_{m}\right)=i T \sum_{n=-\infty}^{+\infty} \int \frac{d^{2} k}{(2 \pi)^{2}} k^{2} \operatorname{tr}\left(\gamma^{0} S\left(i \omega_{n}, k\right) \gamma^{0} S\left(i \omega_{n}+i \nu_{m}, k\right)\right),
$$

It turns out that the thermal conductivity can be given by the discontinuity of this Green function (see 25, 26, 27 for details)

$$
\kappa^{e l}=\frac{1}{4 T} \lim _{\omega \rightarrow 0} \frac{1}{\omega}\left(G\left(p=0, i \nu_{m}=\omega+i 0^{+}\right)-G\left(p=0, i \nu_{m}=\omega-i 0^{+}\right)\right) .
$$

To calculate the Green function in Eq. (20), it is convenient to use the following spectral representation for the fermion thermal Green function 26, 27]

$$
S\left(i \omega_{n}, k\right)=\int_{-\infty}^{+\infty} \frac{d \omega}{2 \pi} \frac{a(\omega, k)}{i \omega_{n}-\omega},
$$

where $a(\omega, k)=2 \operatorname{ImS}\left(i \omega_{n}=\omega-i 0^{+}, k\right)$. After substituting this spectral representation into Eq. (20), one can easily perform the sum over the Matsubara frequencies. Then the result reads

$$
\begin{aligned}
G\left(p=0, i \nu_{m}\right) & =-\frac{i}{2} \int \frac{d^{2} k}{(2 \pi)^{2}} \int \frac{d \omega_{1}}{2 \pi} \int \frac{d \omega_{2}}{2 \pi} \frac{k^{2} \sinh \left(\frac{\omega_{1}-\omega_{2}}{2 T}\right)}{\cosh \left(\frac{\omega_{1}}{2 T}\right) \cosh \left(\frac{\omega_{2}}{2 T}\right)} \\
& \times \frac{\operatorname{tr}\left(\gamma^{0} a\left(\omega_{1}, k\right) \gamma^{0} a\left(\omega_{2}, k\right)\right)}{\left(\omega_{1}-\omega_{2}-i \nu_{m}\right)} .
\end{aligned}
$$

As we see, this representation is indeed very convenient for extracting the discontinuity that is needed for calculation of the thermal conductivity [see Eq. (21)]

$$
\kappa^{e l}=\frac{1}{16 T^{2}} \int \frac{d^{2} k}{(2 \pi)^{2}} \int \frac{d \omega}{2 \pi} \frac{k^{2}}{\cosh ^{2}\left(\frac{\omega}{2 T}\right)} \operatorname{tr}\left(\gamma^{0} a(\omega, k) \gamma^{0} a(\omega, k)\right) .
$$


First, let us calculate the thermal conductivity in the lowest Landau level approximation without taking into account any interactions in the theory. The corresponding fermion Green function is given by

$$
S\left(i \omega_{n}, k\right)=\exp \left(-\frac{k^{2}}{|e B|}\right) \frac{i \omega_{n} \gamma^{0}+m}{\left(i \omega_{n}\right)^{2}-m^{2}}\left(1-i \gamma^{1} \gamma^{2}\right),
$$

so that the spectral density reads

$$
a(\omega, k)=2 \pi \operatorname{sign}(\omega) \exp \left(-\frac{k^{2}}{|e B|}\right) \delta\left(\omega^{2}-m^{2}\right)\left[\omega \gamma^{0}+m\right]\left(1-i \gamma^{1} \gamma^{2}\right) .
$$

Making use of this explicit expression for the spectral density, one obtains the thermal conductivity right away

$$
\kappa^{e l}=\frac{|e B|^{2}}{16 T^{2}} \operatorname{sech}^{2}\left(\frac{m}{2 T}\right) \delta(0) .
$$

This result looks meaningless because of the $\delta$-function in the right hand side which is infinite. Note, however, that this infinity appears due to the $\delta$-like peak of the spectral density $a(k, \omega)$ in Eq. (26). The latter is a direct consequence of our dealing with a free theory. In practice, the spectral density of any physical interacting model is always spread over a finite region of energies.

Instead of actual consideration of the interaction effects on the spectral density in our model, we just modify the latter by applying simple phenomenological arguments. In particular, we introduce the width of quasiparticle states (or in different words, the inverse quantity of the lifetime), $\Gamma \equiv 1 / \tau \ll m$, according to the following rule

$$
\begin{aligned}
\delta\left(\omega^{2}-m^{2}\right) & =\frac{1}{2 m}(\delta(\omega+m)+\delta(\omega-m)) \\
& \rightarrow \frac{1}{2 m \pi}\left(\frac{\Gamma}{(\omega+m)^{2}+\Gamma^{2}}+\frac{\Gamma}{(\omega-m)^{2}+\Gamma^{2}}\right) .
\end{aligned}
$$

At this point we may not specify the nature of the quasiparticle lifetime, but use it as a phenomenological parameter. However, we note that such broadening of the spectral density can be accounted for the scattering of fermions off impurities which always present in real systems [26, 27].

Now we are in a position to estimate the thermal conductivity in our modified theory. In order to preserve the validity of our symmetry breaking analysis due to the magnetic catalysis, we assume that the width $\Gamma$ is considerably smaller than the dynamical mass, $\Gamma \ll m$. Then substitution of the spectral density into the expression for the conductivity (24) leads to the final result

$$
\kappa^{e l} \simeq \frac{|e B|^{2}}{32 \pi T^{2} \Gamma} \operatorname{sech}^{2}\left(\frac{m}{2 T}\right) .
$$


Let us apply this simplified result to the study of thermal conductivity dependence on the magnetic field at a fixed temperature. Qualitatively, we get the following picture. While the magnetic field is weaker than the critical value at a given temperature, there is no energy gap in the spectrum of the model. So, we have to substitute $m\left(T,|e B|<\left|e B_{c}\right|\right)=0$ in Eq. (27). As a result, we observe that the thermal conductivity increases with the magnetic field:

$$
\kappa_{e l}=\frac{|e B|^{2}}{32 \pi T^{2} \Gamma} .
$$

This seems to be just the opposite what the experiment sees [9]. However, we believe that this increase might be the artifact of inappropriate use of the approximation (see below) as well as a result of absence of the Fermi surface in our model and should not be taken as a real prediction.

On the other hand, after the magnetic field gets bigger than the critical value, $|e B|>\left|e B_{c}\right|$, the energy gap $m\left(T,|e B|>\left|e B_{c}\right|\right) \neq 0$ develops and grows with increasing the field strength. So, that gradually, the dependence of the thermal conductivity becomes exponentially damped by the energy gap:

$$
\kappa_{e l}=\frac{|e B|^{2}}{8 \pi T^{2} \Gamma} \cdot \exp \left(-\frac{m(T,|e B|)}{T}\right) .
$$

We expect that this damping of the conductivity is a rather general feature which should be present as soon as an energy gap is developed in the model. We remind that something similar also happens with the thermal conductivity when an ordinary superconducting order parameter develops [26].

In support of the estimates made above, let us comment on the lowest Landau level approximation that was used in the derivation of Eq. (27). Obviously, this approximation could be reliable only if we stay in the region where the dynamical mass is much smaller than the scale of the magnetic field. Let us remind that this condition was indeed satisfied in the case of the magnetic catalysis in QED at zero temperature. While considering finite temperatures, we have to require also that the temperature is much less than the square root of the field strength. This latter requirement gives us one more argument in support of reliability of result for the thermal conductivity (31) in the case of supercritical fields, while adds more distrust in the analysis for subcritical magnetic fields.

Concerning the NJL model, we mention that the ratio $m / \sqrt{|e B|}$, as is seen from Eq. (11), approaches 0.446 in the strong field limit. It is this region that reproduces the square root dependence of the thermal conductivity as a function of the magnetic field. Consider, however, that approximately the same dependence law remains valid down to the values of the ratio $m / \sqrt{|e B|}$ as small as 0.1. Therefore, even though the lowest Landau level approximation may not be perfect in case of the NJL model, it should be reliable enough for the thermal conductivity estimate and for the conclusion about the damping of the latter in the supercritical region. 
The study of the $2+1$ dimensional relativistic NJL model, modeling the dynamics of quasiparticles close to the nodes of the Fermi surface in d-wave superconductors, reveals the dependence for the mass as a function of the magnetic field very close to square root law for rather general assumptions. The appearance of the energy gap is viewed here as a result of breaking a discrete symmetry due to neutral condensate of fermion-antifermion pairs. In condense matter language such a situation could be interpreted as the condensation of electron-hole-like quasiparticle pairs. The results of our paper give an alternative explanation to the recently observed [9] phase transition in cuprites below $T_{c}$.

\section{Acknowledgments}

We would like to thank V. Gusynin and V. Miransky for discussions and pointing our attention to Ref. 13. This work was supported by the Natural Sciences and Engineering Research Council of Canada and in part by the U.S. Department of Energy Grant \#DE-FG02-84ER40153.

\section{References}

[1] V. P. Gusynin, V. A. Miransky, and I. A. Shovkovy, Phys.Rev.Lett.73, 3499 (1994); Phys.Rev.D52, 4718 (1995).

[2] V. P. Gusynin, V. A. Miransky, and I. A. Shovkovy, Phys.Lett.B349, 477 (1995); Phys.Rev.D52, 4747 (1995); Nucl. Phys. B462, 249 (1996).

[3] C. N. Leung, Y. J. Ng, and A. W. Ackley, Phys.Rev.D54, 4181 (1996);

D.-S. Lee, C. N. Leung, and Y. J. Ng, Phys.Rev.D55, 6504 (1997).

[4] D. K. Hong, Y. Kim, and S.-J. Sin, Phys.Rev.D54, 7879 (1996); D. K. Hong, Phys.Rev.D57, 3759 (1998).

[5] V. Elias, D. G. C. McKeon, V. A. Miransky, and I. A. Shovkovy, Phys.Rev.D54, 7884 (1996).

[6] S. P. Klevansky and R. H. Lemmer, Phys.Rev.D39, 3478 (1989).

[7] K. G. Klimenko, Theor. Math. Phys. 89, 1161 (1992); Z. Phys. C54, 323 (1992).

[8] S. Schramm, B. Müller, and A. J. Schramm, Mod. Phys. Lett. A7, 973 (1992).

[9] K. Krishana, N. P. Ong, Q. Li, G. D. Gu, N. Koshizuka, Science 277, 83 (1997). 
[10] S. H. Simon and P. A. Lee, Scaling of the quasiparticle spectrum for $d$-wave superconductors, cond-mat/9611133.

[11] R. B. Laughlin, Magnetic Induction of $d_{x^{2}-y^{2}}+i d_{x y}$ Order in High- $T_{c}$ Superconductors, cond-mat/9709004.

[12] R. B. Laughlin, Physica C234, 280 (1994).

[13] K. Farakos, N. E. Mavromatos, Gauge-theory approach to planar doped antiferomagnets and external magnetic fields, cond-mat/9710288; K. Farakos, G. Koutsoumbas, and N. E. Mavromatos, Dynamical flavor symmetry breaking by a magnetic field in lattice $Q E D$ in three-dimensions, hep-lat/9802037.

[14] G. W. Semenoff and L. C. R. Wijewardhana, Phys.Rev.Lett.63, 2633 (1989).

[15] N. Dorey and N. E. Mavromatos, Nucl. Phys. B368, 614 (1992).

[16] Y. Nambu and G. Jona-Lasinio, Phys. Rev. 122, 345 (1961).

[17] T. Appelquist, M. Bowick, D. Karabali, and L. C. R. Wijewardhana, Phys.Rev.D33, 3704 (1986).

[18] V. L. Berezinsky, Sov. Phys. JETP 32, 493 (1970); J. M. Kosterlitz and D. J. Thouless, J. Phys. C6, 1181 (1973).

[19] V. P. Gusynin, I. A. Shovkovy, Phys.Rev.D56, 5251 (1997).

[20] A. V. Shpagin, Dynamical mass generation in (2+1)-dimensional electrodynamics in external magnetic field, hep-ph/9611412.

[21] N. D. Mermin and H. Wagner, Phys.Rev.Lett.17, 1133 (1966); S. Coleman, Commun. Math. Phys. 31, 259 (1973).

[22] T. Appelquist, D. Nash, and L. C. R. Wijewardhana, Phys.Rev.Lett.60, 2575 (1988).

[23] Y. Hosotani, Phys.Lett.B319, 332 (1993).

[24] R. Kubo, Some aspects of the statistical-mechanical theory of irreversible processes, in "Lectures in Theoretical Physics", V.1, ed. by W. E. Brittin and L. G. Dunham, (Interscience Publishers, New York, 1959).

[25] J. S. Langer, Phys. Rev. 127, 5 (1962).

[26] V. Ambegaokar and L. Tewordt, Phys. Rev. 134, A805 (1964).

[27] V. Ambegaokar and A. Griffin, Phys. Rev. 137, A1151 (1965). 


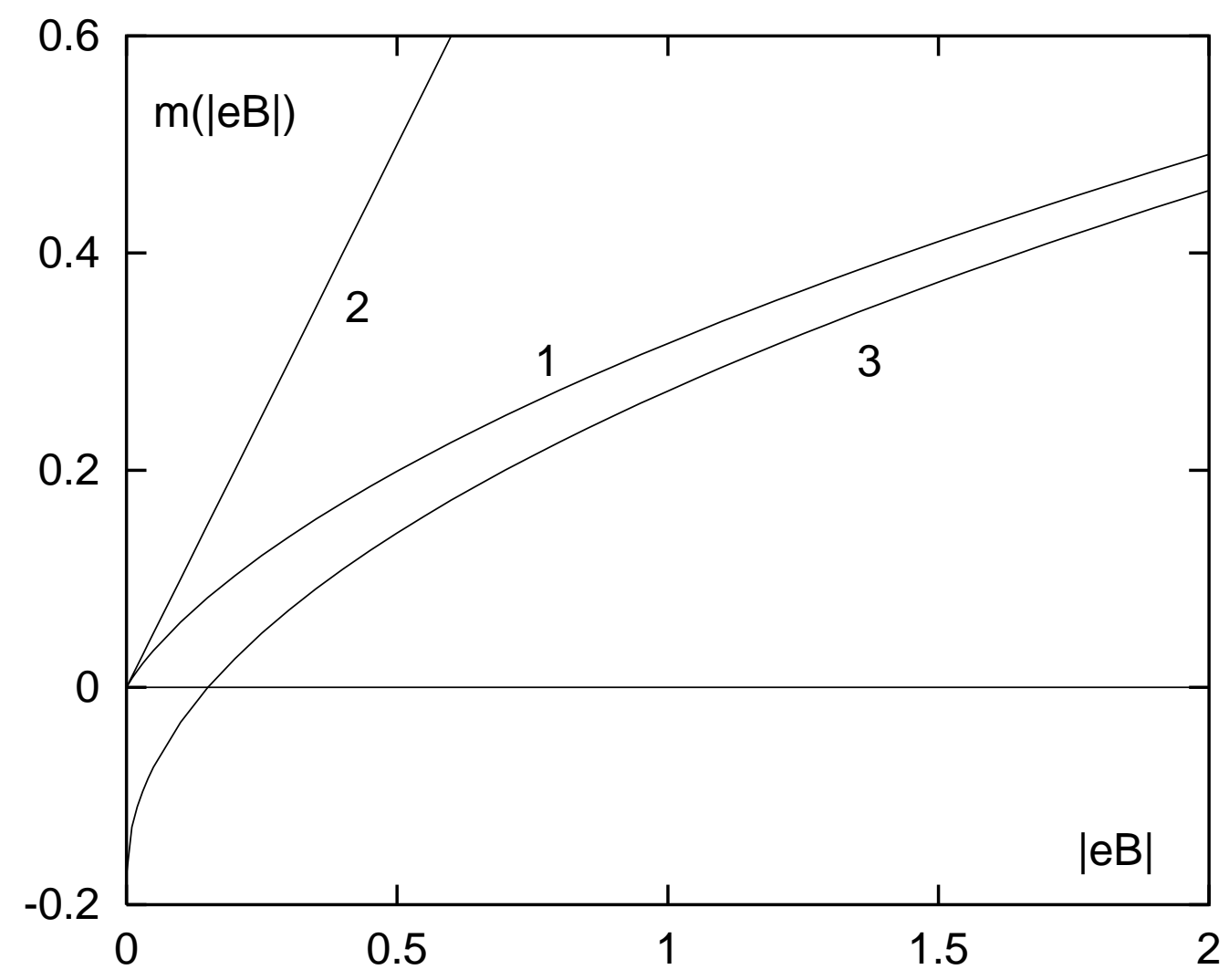

Figure 1: Curve 1 is the numerical solution to the gap equation in the NJL model. Curves 2 and 3 are the weak field and strong field asymptotes of the exact solution, respectively. All quantities are measured in units of $\mu$. 